All course materials, includ-

ing the original lecture, are

available as webcasts/pod-

casts at www.ers-education.

org/lsc2009.htm

\title{
The pathogenesis of pre-invasive lesion development
}

E. Brambilla

Laboratoire de Pathologie Cellulaire

Dept of Pathology

CHU Albert Michallon

University Joseph Fourier

Grenoble

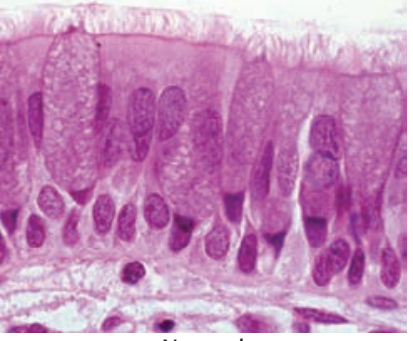

Normal

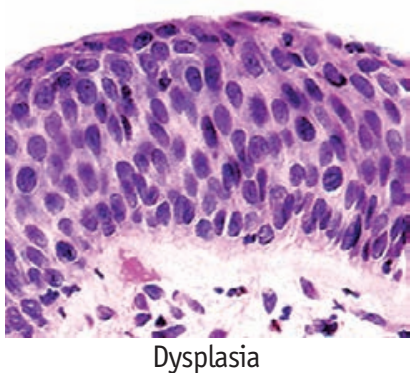

\section{Educational aims}
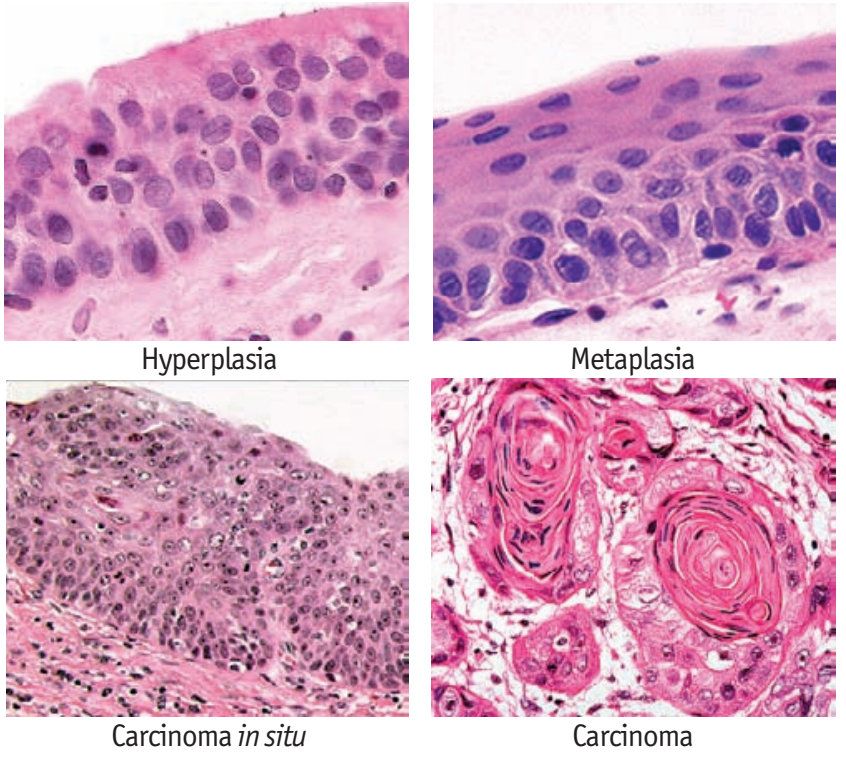

Metaplasia

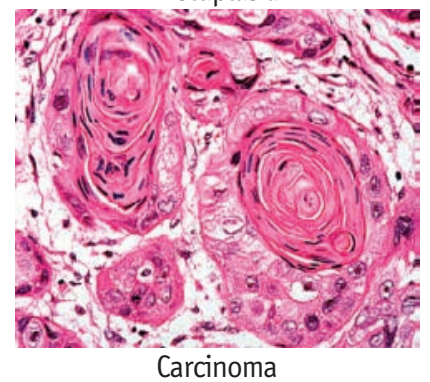

Carcinoma

France

EBrambilla@chu-grenoble.fr

Provenance

Adapted from an ERS School Course.

\section{Competing interests}

None declared.

1 To describe the progression of pre-invasive bronchial lesions.

1 To highlight malignant transformation causes and risks.

1 To explain somatic and epigenetic abnormalities contributing to lesions.

\section{Summary}

The progression of pre-invasive bronchial lesions is a multifocal and multistep process. Morphological malignant transformation is driven by the accumulation of multiple genetic and epigenetic abnormalities, the rate and number of which determines the risk and rate of progression toward cancer. The field effect of mutagenesis in airways is characterised by the multifocality of the lesions. To date, no evidence has been presented for genetic alterations accumulating in a known sequence. No individual alteration is sufficient to predict the occurrence of invasive cancer in the bronchial tree.

Lung cancer is the leading cause of death from cancer worldwide. The high mortality rate (80-85\% within 5 years) results, in part, from a lack of effective tools to diagnose the disease at an early stage. Given that cigarette smoke creates a field of injury throughout the airways, epithelial malignancies, including bronchial epithelial malignancies, arise after a series of progressive pathological changes including hyperplasia, different grades of dysplasia and carcinoma in situ (CIS) [1]. These pre-malignant changes can accompany cancer 
or occur in the normal epithelium of individuals at high risk.

Approximately $85 \%$ of lung cancer cases are related to the use of tobacco [2]. Our knowledge of genetic predisposition for lung cancer has recently been expanded with the discovery of acetylcholine receptor genes (A3, A5 and B4), which have high-susceptibility variants for lung cancer. These acetylcholine receptors activate an autocrine loop driving proliferation and invasion at the level of bronchial epithelial cells. This is the first definite link between a polymorphism at a specific gene and pathogenesis $[3,4]$. Other risk factors for lung cancer include chronic obstructive pulmonary disease and peripheral arterial disease.

\section{Development of invasive tumours}

Bronchial epithelial cells undergo a long series of morphological changes to become invasive tumours (figure 1). During this transformation there is an accumulation of somatic mutations (chromosome deletion, methylation and point mutations) that disrupt key factors and pathways of cell proliferation and apoptosis. Somatic mutation starts before the pre-neoplastic stage, coinciding with transformations at the morphological level. During the pre-invasive stage, there is a strong involvement of autocrine and paracrine effects on cell proliferation in addition to other paracrine and autocrine loops taking place during the passage from CIS to invasion. At the primary invasive and metaplastic stage, somatic deregulation causes changes in matrix proteases, adhesion-migration and angiogenesis.

The morphological steps from normal bronchial epithelium to carcinoma cells have been

Steps of morphological changes for bronchial lesions.

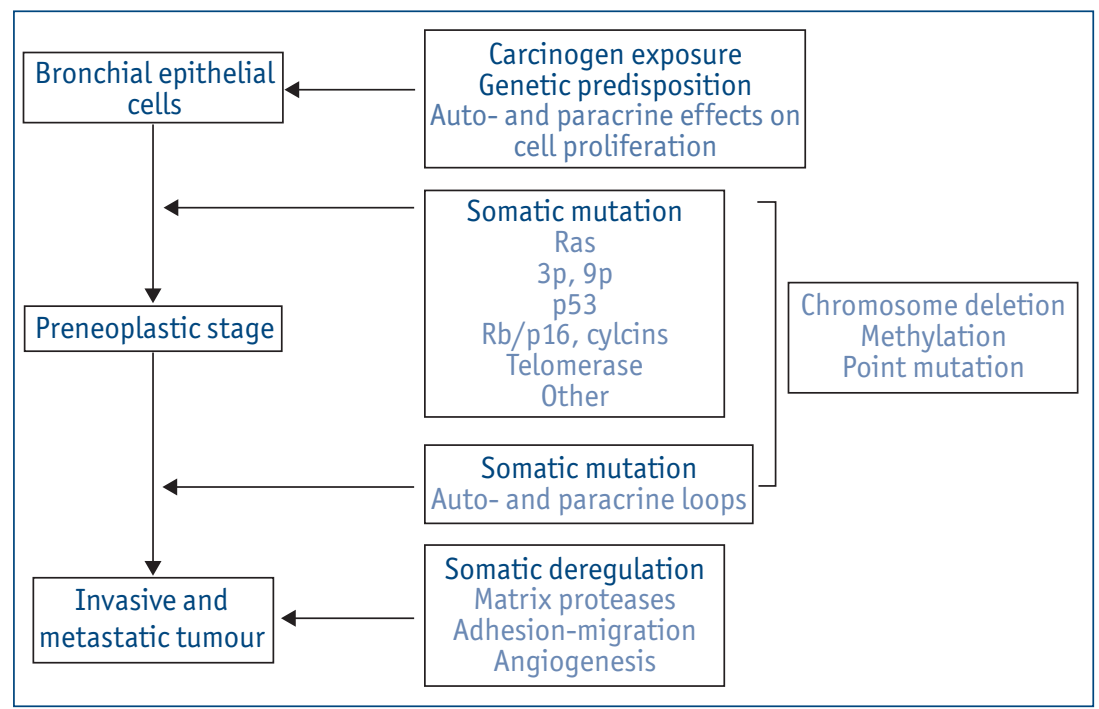

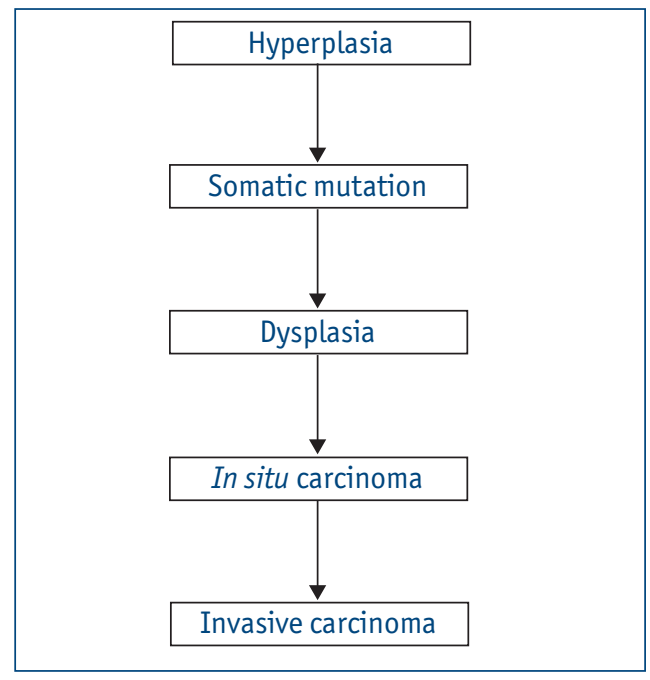

\section{Figure 2}

Stages in the progression of bronchial lesions.

identified (figure 1). In normal epithelium, basal cells (the progenitor cells for squamous carcinoma and probably small cell carcinoma) can be seen, along with ciliated and goblet cells. In hyperplasia, polystratification of the basal cell line occurs. In metaplasia, a complete transformation of the glandular epithelium to squamous epithelium can be seen. There are mild, moderate and severe levels of dysplasia; in severe dysplasia, which directly precedes $\mathrm{CIS}$, the orientation and maturation of cells is difficult to see. When the bronchial epithelium reaches the $\mathrm{CIS}$ stage it will almost certainly evolve into carcinoma.

With well-identified morphological stages of lesion progression, the main problem is predicting the progression from one morphological stage to the next. In total, $<10 \%$ of metaplasias and $<40 \%$ of mild or moderate dysplasias will become cancer. The only thing researchers are sure of is that CIS will lead to the development of an invasive carcinoma even with long-term local treatment. Therefore it is clear that molecular identity, cell-cycle regulation, apoptosis/ senescence and DNA damage checkpoints are important factors to identify at all morphological stages in order to improve the prediction of preinvasive lesions evolving into lung cancer.

\section{Field carcinogenesis}

Sequential molecular abnormalities in the pathogenesis of epithelial bronchial tumours indicate that genetic changes commence in histologically normal epithelium, progress with increasing degrees of epithelial changes, follow a sequence from early to late stages, and are extensive and multifocal throughout the epithelium, indicating 


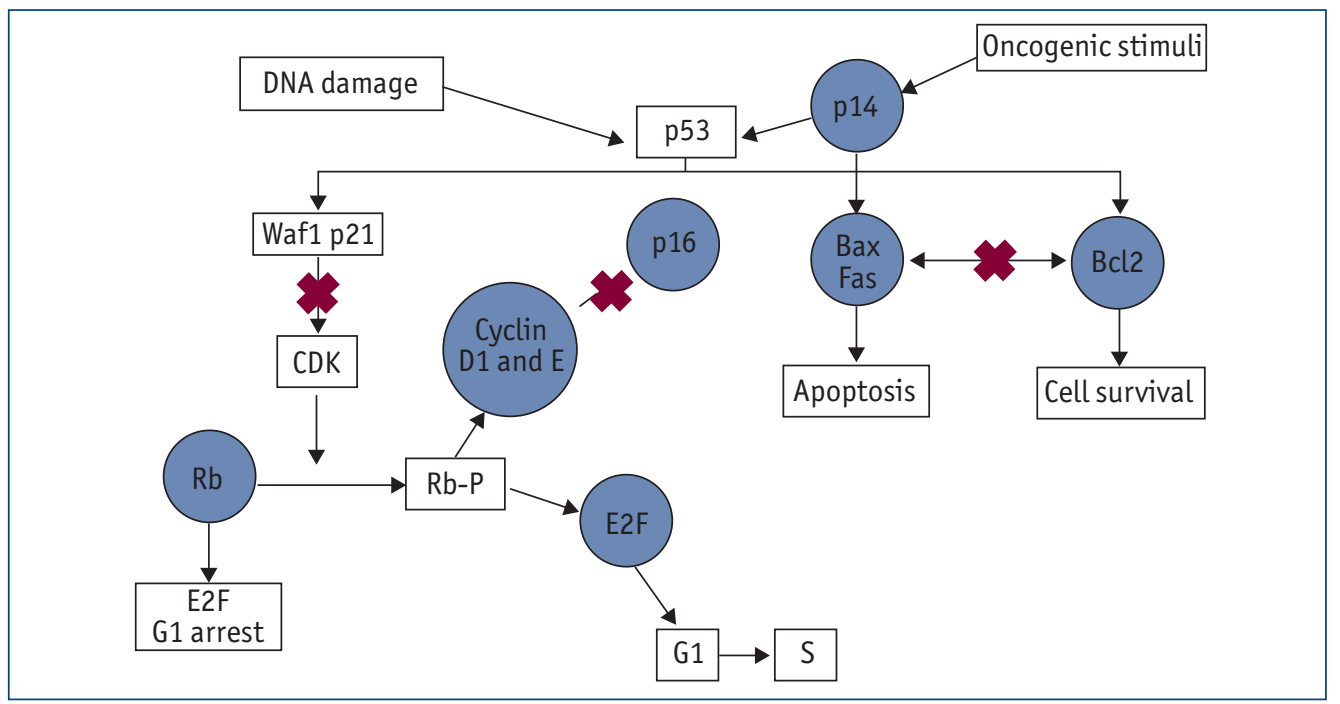

a field effect known as "field cancerisation" [5]. Each lesion is independent from all others and has a multistep process of progression at the molecular level which depends on the number, accumulation rate and chronology of genetic alterations. The time sequence of progression differs from patient to patient.

Multiple clonal and subclonal patches of molecularly abnormal epithelial cells with or without morphological changes can be detected throughout the affected epithelium, specifically in smokers. Even histologically normal and $a$ fortiori dysplastic lesions may show a number of somatic mutations of oncogene and tumour suppressor genes. Allelic loss (loss of heterozygosity, methylation of $\mathrm{CpG}$ islands and point mutations) of chromosomes 3p, 9p21 and 17p13 are common in smokers' epithelial cells $[6,7]$. A p53 mutation is usually one of the first mutations to be seen. Promoter methylation is extremely frequent in smokers without cancer and occurs during very early metaplasia. Increased telomerase activity is also very frequent in smokers. Furthermore, genome-wide expression profiles that can detect cancerisation at a distance from the lesion have been identified [8].

Although the risk of progression increases from metaplasia to the successive grades of dysplasia and CIS, no clear prediction can be based on morphological grading alone. Knowledge of the molecular identity is necessary to evaluate the risk of progression.

\section{p53 pathway}

The key pathway altered in pre-invasive bronchial lesions (excluding those preceding adenocarcinoma) is the p53 signalling pathway of cell cycle control and apoptosis (figure 3). p53 is a transcription factor that acts as a sensor of stress responding to oncogenic stimuli, DNA damage and hypoxia. p53 transcription targets downstream genes involved in cell cycle arrest, apoptosis or DNA repair and upstream regulatory genes including p14 and mdm2. p53 is the most frequently mutated gene in lung cancer.

Stabilising p53 mutations are easy to detect in immunohistochemistry since the mutant protein has a long half-life, of up to $10 \mathrm{~h}$, in contrast to the normal protein's half-life of $7 \mathrm{~min}$. A p53 mutation may be seen as early as mild dysplasia. The presence of a p53 mutant phenotype is clearly linked to the progression of preinvasion to invasion $[9,10]$.

\section{Rb pathway}

A downstream signalling pathway of $p 53$ involves the retinoblastoma protein, $\mathrm{Rb}$, which plays a key role in mitochondrial apoptosis (figure 4). The $\mathrm{Rb}$ pathway mediates $\mathrm{Gl}$ arrest through the cyclindependent kinase (CDK) inhibitor p21. Unphosphorylated Rb binds to E2F1 and suppresses its transcriptional activity in G1-S transition. The protein complexes CDK6-cyclin E and CDK4-cyclin D1 phosphorylate $\mathrm{Rb}$ by releasing E2F1 during G1-S transition under the effect of growth factors or oncogenic stimuli. Therefore both the loss of and the hyperphosphorylation of the $\mathrm{Rb}$ protein disrupt the G1 checkpoint and are common in lung cancer.

The main causes of inactivation of $\mathrm{Rb}$ are through the overexpression of cyclin D1 or E or loss of p16 expression (figure 5) [10, 12]. Loss of p16 expression has been seen in 12\% of moderate dysplasia and $30 \%$ CIS cases [13]. In patients with cancer, overexpression of cyclin D1 has been shown to occur in $6 \%$ of hyperplasia

\section{Figure 3}

The p53 pathway maintains genomic stability by arresting or eliminating damaged cells. For example, DNA damage activates p53 which turns on p21 transcription. $p 21$ then binds to and inhibits cyclin-dependent kinases (CDKs), leading to hypophosphorylation of retinoblastoma protein $(R b)$. This prevents the release of E2F and blocks the G1 to S transition. p53 also acts through Bax and Fas to cause apoptosis and Bcl2 to halt cell survival. 


\section{Figure 4}

The $R b$ pathway. $R b$ is the main downstream effector of $p 53$ in the control of G1 arrest. $R b$ function depends on the level of phosphorylation, which is achieved by CDK 2 and 4 in complex with either cyclin $E$ or cyclin D1. These kinase complexes are retro-controlled by the CDK inhibitors p21 (the transcriptional target of p53), p16 (INK4b) and p27 (kip1). Phosphorylated Rb releases $E 2 F 1$, which disrupts the $G 1$ checkpoint. Loss of functional CDK inhibitors such as p53, activation/ gain of cyclin D1 or cyclin E, and Rb loss all result in E2F1 activation and disruption of the G1 checkpoint. Adapted from [11].

\section{Figure 5}

Cyclin D1 and E overexpression in pre-invasive bronchial lesions.

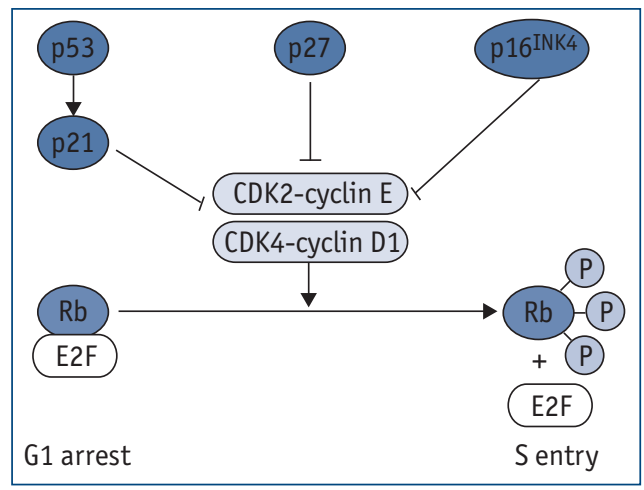

and metaplasias, $17 \%$ of mild dysplasias, $46 \%$ of moderate dysplasias and 38\% of CIS [13]. Cyclin $\mathrm{E}$ overexpression has been demonstrated to occur in nonsmall cell lung cancer (NSCLC) [14]. The overexpression of cyclin D1 and $\mathrm{E}$ is easy to detect immunohistochemically in pre-invasive lesions and occurs with increasing intensity and frequency with the severity of pre-invasive lesions from metaplasia to CIS.

\section{Bcl2 and Bax}

Escaping apoptosis is a major process in the development of tumours. Key factors in mitochondrial apoptosis are anti-apoptotic Bcl2 and proapoptotic Bax. A distinct profile of expression of $\mathrm{Bax}$ and $\mathrm{Bcl} 2$ has been found in neuroendocrine lung tumours: in low-grade tumours, there is a predominance of $\mathrm{Bax}$, while in high-grade tumours Bcl2 predominates [15]. These results suggest that the aggressiveness of neuroendocrine tumours could be linked to apoptosis-related factors, in addition to proliferation.

In most small cell lung cancers (SCLC) Bcl2 is elevated in contrast to Bax which is downregulated in SCLC. This results in a Bcl2:Bax ratio of $>1$ in $95 \%$ of SCLC making cells prone to

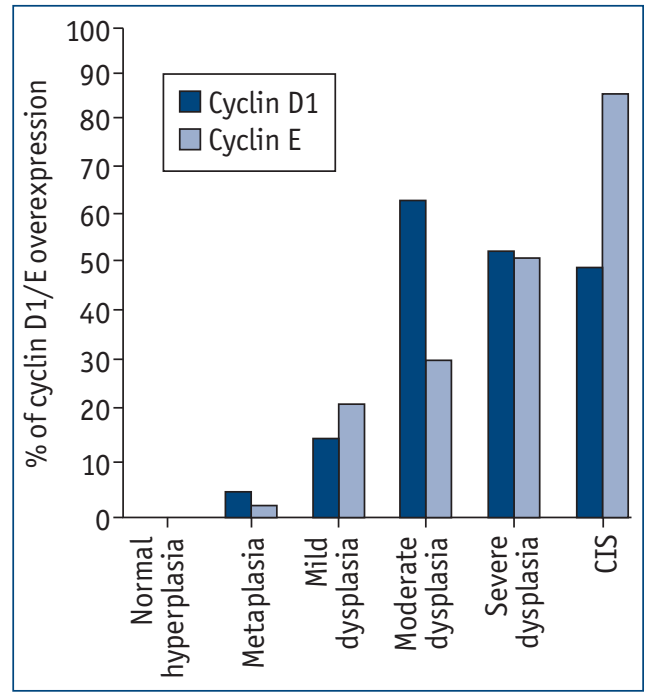

resistance to apoptosis [15]. This ratio is reversed towards Bcl2 during mild dysplasia [9], suggesting an early avoidance of apoptosis during the pre-neoplastic process.

\section{Accumulation of abnormalities}

The accumulation and number of these molecular abnormalities regarding cell cycle deregulation, loss of G1-G2 checkpoints and escape from apoptosis (p53 stabilisation, cyclin D1 and cyclin $\mathrm{E}$ overexpression and the alteration of the Bax:Bcl2 ratio) collectively constitute a risk factor for progression from the pre-invasion state to lung cancer [10]. There is a large difference between the number of abnormalities seen in lesions at different morphological stages of progression. In severe dysplasia, a large proportion of patients have more than three of the above abnormalities, compared with only a small number seen in mild dysplasia. This suggests that molecular pathology could be used to select a subpopulation of patients at high risk of developing of CIS or lung cancer.

\section{Telomerase}

Telomerase is a reverse transcriptase that synthesises telomeric sequences. The presence of telomerase is an important marker for cell immortality in malignancies because it maintains the length of telomeres. Telomeres are repetitive sequences located at the end of chromosomes that prevent end-toend fusion and exonuclease excision. Telomeres shorten after cell division, limiting the life span of cells. When telomeres become critically short, the p53 pathway is activated and the cell will normally enter apoptosis or senescence. In tumour cells, this M1 stage is rescued by the inactivation of the $\mathrm{p} 53 / \mathrm{Rb}$ pathway. Telomerase is reexpressed in tumour cells lacking the p53 and Rb-mediated checkpoints. Telomerase contains hTERT, a protein catalytic subunit, which is the limiting factor for telomerase activity. The upregulation of telomerase at this stage is thought to represent the early steps of immortalisation in tumorigenesis [16].

In pre-invasive bronchial lesions, hTERT expression is significantly increased in line with the severity of their grade $[17,18]$. Nearly $100 \%$ of SCLCs have detectable or high levels of telomerase [19]. Telomere attrition reaches its maximum level at squamous metaplasia, as telomeres shorten following several cycles of all cell division, but further increase in telomere length as well as telomerase activation follows along the 
progression from early to late preinvasive lesions and invasion. Telomeres are the shortest length at the metaplasia stage, which is the initiation of cancer progression: after this point telomerase activation occurs encouraging tumour progression [17]. The squamous metaplasia phase may represent a M1 stage preceding $\mathrm{p} 53 / \mathrm{Rb}$ inactivation and telomerase activation.

\section{DNA damage response}

Commonly expressed markers of activated DNA damage have been seen in different stages of lung cancers. These include phosphorylated H2AX and CHK2. H2AX and CHK2 are key regulators in DNA damage checkpoints and the phosphorylation of these factors causes cells to enter into apoptosis. In normal bronchi, the level of phosphorylated H2AX is much lower than that seen in severe dysplasia, demonstrating that DNA damage is extremely high even in early precursor lesions $[20,21]$. The level of phosphorylated H2AX increases steadily from normal bronchi to the CIS stage. Mutations in the DNA damage response pathway may also allow cell proliferation, genomic instability and tumour progression [20].

\section{Epigenetic modification}

Epigenetic modifications are molecular mechanisms that regulate gene expression without changing the DNA sequence. These include the following. 1) The alteration of the methylation status of DNA within CpG islands leads to the silencing of tumour suppressor genes or the activation of oncogenes. 2) Covalent modifications of histone tails cause chromatin remodelling. 3) MicroRNAs lead to mRNA silencing and loss of protein expression.

\section{Aberrant methylation}

DNA methylation is a normal process that occurs as part of a complex chromatin network influenced by modifications in histone structure. Changes in the pattern of DNA methylation have been shown to be a common event in human cancer and cause abnormalities of gene expression, chromosome structure, timing of DNA replication and chromatin organisation.

DNA methylation is restricted to " $\mathrm{CpG}$ islands" which, in expressed genes, are unmethlyated. Aberrant methylation of $\mathrm{CpG}$ islands located near the promoter region of these genes causes gene silencing. In lung cancer, as many as 100 genes have been found to be silenced by promoter methylation [22]. Methylation occurs in the early stages of lung cancer [23] and if it is detected in the sputum of smokers with dysplasia it has been shown to be a predictor of cancer development [24]. DNA methylation may also predict reoccurrence in stage I NSCLC [25]. A study examining methylation in eight genes in primary NSCLC and corresponding non-malignant tissue found that at least one gene was methylated in $85 \%$ of NSCLC [26]. These data demonstrate that methylation is the most common mechanism to inactivate cancer-related genes in NSCLC.

\section{Histone modifications}

The acetylation and methylation of lysine residues on histone tails plays a crucial role in regulating chromatin packaging, nuclear architecture, gene expression and genomic stability [27-32]. In lung cancer cells, hypermethylation of $\mathrm{CpG}$ islands in promoter regions of tumour suppressor genes has been associated with particular combinations of histone markers. For example, deacetylation of histones $\mathrm{H} 3$ and $\mathrm{H} 4$, loss of trimethylation of histone $\mathrm{H} 3$ lysine 4 and an increase in trimethylated H3K9 and H3K27 have been seen in cancer cell lines.

Cancer cells have been demonstrated to have a loss of monoacetylated and trimethylated forms of histone $\mathrm{H} 4$ [32]. These changes in $\mathrm{H} 4$ occur early and accumulate as the tumour progresses. The data suggest that the global loss of monoacetylated and trimethylated forms of $\mathrm{H} 4$ is a hallmark of tumour cells in humans.

Epigenetic modifications of $\mathrm{H} 4$ have also been studied in 157 lung carcinomas using antibodies specific to lysines $\mathrm{K} 5, \mathrm{~K} 8, \mathrm{~K} 12, \mathrm{~K} 16$ and trimethylated H4K20 [33]. Compared with normal lung, the cancer cells showed hyperacetylation of $\mathrm{H} 4 \mathrm{~K} 5 / \mathrm{H} 4 \mathrm{~K} 8$, hypoacetylation of $\mathrm{H} 4 \mathrm{~K} 12$ / H4K16 and a loss of H4K20 trimethylation. The loss of trimethylated H4K20 can be seen as early as pre-neoplastic bronchial lesion and is frequent in squamous cell carcinoma. The reduction increases with the grade of pre-invasive lesion. Therefore, H4K20 could be a potential candidate biomarker for the early detection of therapeutic approaches to lung cancer.

\section{Conclusion}

A full array of molecular abnormalities leading to intrinsic proliferation, cell cycle check 
points disruption, escape of apoptosis, cell immortalisation and epigenetic modification are the leading forces of progression of malignant transformation, particularly in smokers.
At any stage of the morphological scale, the number and accumulation of abnormalities of key signalling pathways represent predictive factors for invasion.

\section{References}

1. Travis W, Brambilla E, Muller-Hermelink HK, Harris CC. World Health Organization Classification of Tumours. Pathology and Genetics of Tumours of the Lung, Pleura, Thymus and Heart. IARC Press, Lyon, 2004.

2. Auerbach 0, Stout AP, Hammond EC, Garfinkel J. Changes in bronchial epithelium in relation to cigarette smoking and in relation to lung cancer. N Engl J Med 1961; 265: 253-267.

3. Thorgeirsson TE, Geller F, Sulem P, et al. A variant associated with nicotine dependence, lung cancer and peripheral arterial disease. Nature 2008; 452: 638-642.

4. Hung RJ, McKay JD, Gaborieau V, et al. A susceptibility locus for lung cancer maps to nicotinic acetylcholine receptor subunit genes on 15q25. Nature 2008; 452: 633-637.

5. Wistuba II, Meyerson M. Chromosomal deletions and progression of premalignant lesions: Less is more. Cancer Prev Res 2008; 1: 404-408.

6. Mao L, Lee JS, Fan YH, et al. Frequent microsatellite alterations at chromosomes 9p21 and 3p14 in oral premalignant lesions and their value in cancer risk assessment. Nat Med 1996; 2: 682-685.

7. Wistuba, II, Lam S, Behrens C, et al. Molecular damage in the bronchial epithelium of current and former smokers. J Natl Cancer Inst 1997; 89: 1366-1373.

8. Spira A, Beane JE, Shah V, et al. Airway epithelial gene expression in the diagnostic evaluation of smokers with suspect lung cancer. Nat Med 2007; 13: 361-366.

9. Brambilla E, Gazzeri S, Lantuejoul S, et al. p53 mutant immunophenotype and deregulation of p53 transcription pathway (Bcl2, Bax, and Waf1) in precursor bronchial lesions of lung cancer. Clin Cancer Res 1998 ; 4: 1609-1618.

10. Jeanmart $M$, Lantuejoul $S$, Fievet $F$, et al. Value of immunohistochemical markers in preinvasive bronchial lesions in risk assessment of lung cancer. Clin Cancer Res 2003; 9: 2195-2203.

11. Brambilla E, Gazdar A. Pathogenesis of lung cancer. Signaling pathways - roadmap for therapies. Eur Respir J 2009; 33: 1485-1497.

12. Brambilla E, Moro D, Gazzeri S, Brambilla C. Alterations of expression of Rb, p16(INK4A) and cyclin D1 in non-small cell lung carcinoma and their clinical significance. J Pathol 1999; 188: 351-360.

13. Brambilla E, Gazzeri S, Moro D, Lantuejoul S, Veyrenc S, Brambilla C. Alterations of Rb pathway (Rb-p16INK4-cyclin D1) in preinvasive bronchial lesions. Clin Cancer Res 1999; 5: 243-250.

14. Lonardo F, Rusch V, Langenfeld J, Dmitrovsky E, Klimstra DS. Overexpression of cyclin D1 and E is frequent in bronchial preneoplasia and precedes squamous cell carcinoma development. Cancer Res 1999; 59: 2470-2476.

15. Brambilla E, Negoescu A, Gazzeri S, et al. Apoptosis-related factors p53, Bcl2, and Bax in neuroendocrine lung tumors. Am J Pathol 1996; 149: 1941-1952.

16. Meyerson M. Role of telomerase in normal and cancer cells. J Clin Oncol 2000; 18: 2626-2634.

17. Lantuejoul S, Soria JC, Morat L, et al. Telomere shortening and telomerase reverse transcriptase expression in preinvasive bronchial lesions. Clin Cancer Res 2005; 11: 2074-2082.

18. Hiyama K, Hiyama E, Ishioka S, et al. Telomerase activity in small-cell and non-small-cell lung cancers. J Natl Cancer Inst 1995; 87: 895-902.

19. Lantuejoul S, Soria JC, Moro-Sibilot D, et al. Differential expression of telomerase reverse transcriptase (hTERT) in lung tumours. Br J Cancer 2004; 90: 1222-1229.

20. Bartkova J, Horejsi Z, Koed K, et al. DNA damage response as a candidate anti-cancer barrier in early human tumorigenesis. Nature 2005; 434: 864-870.

21. Gorgoulis VG, Vassiliou LV, Karakaidos P, et al. Activation of the DNA damage checkpoint and genomic instability in human precancerous lesions. Nature 2005; 434: 907-913.

22. Shames DS, Girard L, Gao B, et al. A genome-wide screen for promoter methylation in lung cancer identifies novel methylation markers for multiple malignancies. PLoS Med 2006; 3: e486.

23. Zochbauer-Muller S, Lam S, Toyooka S, et al. Aberrant methylation of multiple genes in the upper aerodigestive tract epithelium of heavy smokers. Int J Cancer 2003; 107: 612-616.

24. de Fraipont F, Moro-Sibilot D, Michelland S, Brambilla E, Brambilla C, Favrot MC. Promoter methylation of genes in bronchial lavages: a marker for early diagnosis of primary and relapsing non-small cell lung cancer? Lung Cancer 2005; 50: 199-209.

25. Brock MV, Hooker CM, Ota-Machida E, et al. DNA methylation markers and early recurrence in stage I lung cancer. $N$ Engl J Med 2008; 358: 1118-1128.

26. Zochbauer-Muller S, Fong KM, Virmani AK, Geradts J, Gazdar AF, Minna JD. Aberrant promoter methylation of multiple genes in non-small cell lung cancers. Cancer Res 2001; 61: 249-255.

27. Gibbons RJ. Histone modifying and chromatin remodelling enzymes in cancer and dysplastic syndromes. Hum Mol Genet 2005; 14 Spec No 1: R85-R92.

28. Esteller M. Cancer epigenomics: DNA methylomes and histone-modification maps. Nat Rev Genet 2007; 8: 286-298.

29. Jenuwein T, Allis CD. Translating the histone code. Science 2001; 293: 1074-1080.

30. Groth A, Rocha W, Verreault A, Almouzni G. Chromatin challenges during DNA replication and repair. Cell 2007; 128: 721-733.

31. Kouzarides T. Chromatin modifications and their function. Cell 2007; 128: 693-705.

32. Fraga MF, Ballestar E, Villar-Garea A, et al. Loss of acetylation at Lys 16 and trimethylation at Lys 20 of histone $\mathrm{H} 4$ is a common hallmark of human cancer. Nat Genet 2005; 37: 391-400.

33. Van Den Broeck A, Brambilla E, Moro-Sibilot D, et al. Loss of histone H4K20 trimethylation occurs in preneoplasia and influences prognosis of non-small cell lung cancer. Clin Cancer Res 2008; 14: 7237-7245. 